\title{
THE EFFICACY OF THE M-HEALTH PARADIGM: INCORPORATING TECHNOLOGICAL, ORGANISATIONAL AND MANAGERIAL PERSPECTIVES
}

\author{
Ashish N. Dwivedi, Rajeev K. Bali, Raouf N.G. Naguib, and Nahy S. \\ Nassar $^{*}$
}

\section{THE ROLE OF TECHNOLOGY IN TELEHEALTH}

Technology strongly influences the way we work and is creating opportunities and new demands for a range of different approaches to telehealth (Feldman and Gainey, 1997). Telecommunications have evolved and have been accompanied by an evolution in attitudes to information and communications technologies (Stanworth, 1998). Previously, only companies owned computers and it was the IT specialists, rather than ordinary users, who determined their use and application. Today's response to technological change is profoundly different. On average, around 1 in 4 European households already owns a personal computer; in some countries this rises to more than $50 \%$ and in some local communities it is even higher.

A recent study confirms this trend and predicts that, in two years time, it is expected that the use of information communication technology will increase markedly (Marien, 1989). The ease with which we use them and the take-up of remote working in the European Union continues at a rapid pace. Recent estimates (European Telework Organization, 1999) show that approximately 6.7 million Europeans $(4.5 \%$ of the workforce) were practising remote working in one form or another at the beginning of 1999.

Social, cultural, economic and regulatory factors determine how we organise our business, our work and, hence, our lives (Stanworth, 1998). Technology-led change opens up opportunities for new working methods in three main ways: allowing existing activities to be carried out more rapidly, with more consistency and at a lower cost than could previously be achieved.

\footnotetext{
${ }^{*}$ Rajeev K. Bali, BIOCORE, School of Mathematical and Information Sciences, Coventry University, UK.
} 
Today, the explosive growth of the Internet has promoted the trend for investment in information and communication devices and the healthcare industry is an active participant in this trend (Kazman and Westerheim, 1999). It would be fair to state that advances in communications technology are dramatically changing the delivery of healthcare services (Schooley, 1998).

\section{THE HEALTHCARE SECTOR}

Modern healthcare is the largest sector of the US economy (Kazman and Westerheim, 1999). However, IT expenditure in healthcare organisations, as a portion of revenues, is in the region of $2 \%$, far below the $7-10 \%$ mark in other information-intensive industries (Moran, 1998). HIs are demanding healthcare applications that offer a number of utilities whilst they themselves allocate only a very small component of the total funds at their disposal (see table 1) (Morrissey, 2000, 2001 and 2002).

Analysts are confident that the above situation is about to undergo a sea change. Investor confidence in technology growth in healthcare is so strong that, between 1992 to 1996, there was a quintuple leap in the number of publicly traded health information technology companies. In 1998, the top 35 companies had market capitalization of over \$25 billion (Moran, 1998). In 1999, about 43\% of US-based Internet users used the Web to locate healthcare related information (Kazman and Westerheim, 1999). This clearly indicates that e-healthcare and its applications (such as m-health) are here to stay.

\subsection{Budgeted Expenditure on IT in Healthcare Institutions and Healthcare Trends}

An analysis (see Table 1) of the Budgeted IT expenditure (as a ratio of the information systems budget to the total operating expenses) shows that some of the major technologies that have showed a lot of promise are Workflow Management Systems, Mobile Computing technologies such as Personal Digital Assistant (PDA), Wireless Local Area Network (WLAN) and Object Oriented Technology (Dwivedi et al, 2001a, 2001b; 2002).

\subsection{The Role of Telecommunications Technology in Health}

The changes in information technology, particularly in the telecommunications technology have brought about fundamental changes throughout the healthcare process (Applebaum and Wohl, 2000). The change process undergone is confirmed by research which states that in the period 1997-2000, 85\% of healthcare organizations have undergone some sort of transformation (Sherer, 1995).

One of the most important technological changes in electronics has been the ability to convert signals from an analogue to a digital medium (images or signals are converted into digital code by using an analogue-to-digital conversion device) - a process referred to as Digitization (Wallace, 1997). Digitization in healthcare has meant that it is now possible to take healthcare related information in different formats (audio, video, sound) and deliver the same at high speeds in the same basic format. 
Table 1. Modern Healthcare's annual survey of information system trends in the healthcare industry Adapted from (Morrissey, 2000, 2001 and 2002)

\begin{tabular}{|c|c|c|c|}
\hline Year & 2000 & 2001 & 2002 \\
\hline $\begin{array}{l}\text { No of healthcare } \\
\text { organizations } \\
\text { surveyed }\end{array}$ & $\begin{array}{l}224 \text { healthcare } \\
\text { organizations }\end{array}$ & $\begin{array}{l}212 \text { healthcare } \\
\text { organizations }\end{array}$ & $\begin{array}{l}255 \text { healthcare } \\
\text { organizations }\end{array}$ \\
\hline $\begin{array}{l}\text { Budgeted IT } \\
\text { expenditure } \\
\text { (Ratio of the } \\
\text { information } \\
\text { systems budget to } \\
\text { the total operating } \\
\text { expenses) }\end{array}$ & $\begin{array}{l}\text { Average IT expenditure } \\
\text { at } 2.6 \% \\
\text { Over } 50 \% \text { put it } \\
\text { between } 2 \% \text { and } 4 \%\end{array}$ & $\begin{array}{l}\text { Average IT expenditure } \\
\text { at } 2.53 \% \\
25 \% \text { spent about } 1.5 \% \\
\text { on IT whilst } 60 \% \text { are } \\
\text { spent } \\
\text { around } 2.5 \%\end{array}$ & $\begin{array}{l}\text { Average IT expenditure } \\
\text { at } 2.56 \% \\
\text { More than } 50 \% \text { spent } \\
\text { between } 2 \% \text { and } 3.5 \%\end{array}$ \\
\hline $\begin{array}{l}\text { Information } \\
\text { systems (IS) } \\
\text { priorities }\end{array}$ & $\begin{array}{l}\text { Internet and intranets - } \\
\text { priority } \\
\text { Clinical information } \\
\text { systems (CIS) tops the IS } \\
\text { priority } \\
\text { General-accounting } \\
\text { software was the } \\
\text { predominant application } \\
\text { that was being used on the } \\
\text { Intranet }\end{array}$ & $\begin{array}{l}65 \% \text { considered CIS as } \\
\text { the most important IS } \\
\text { priority } \\
73 \% \text { were not willing to } \\
\text { outsourcing clinical } \\
\text { information systems and } \\
\text { services }\end{array}$ & $\begin{array}{l}71 \% \text { considered CIS as } \\
\text { the most important IS } \\
\text { priority } \\
\text { Need felt to improve IS } \\
\text { for better clinical } \\
\text { communication for } \\
\text { physicians } \\
80 \% \text { - No interest in } \\
\text { outsourcing clinical } \\
\text { information systems } \\
\text { and services }\end{array}$ \\
\hline $\begin{array}{l}\text { Clinical Use of } \\
\text { Web technology } \\
\text { (Intranets) }\end{array}$ & $\begin{array}{l}60 \% \text { - felt that could IT } \\
\text { could facilitate data } \\
\text { exchange among } \\
\text { caregivers i.e. physician } \\
\text { ordering of tests and access } \\
\text { to test results }\end{array}$ & $\begin{array}{l}\text { Low interest in } \\
\text { maintaining a patient's } \\
\text { personal health record } \\
\text { accessible via the WWW } \\
\text { and matching patients } \\
\text { with clinical research. } \\
\text { However there is } \\
\text { renewed importance of } \\
\text { addressing changes in this } \\
\text { area due to regulatory } \\
\text { obligations }\end{array}$ & $\begin{array}{l}\text { Despite acknowledging } \\
\text { that medication interaction } \\
\text { and dosing alerts are } \\
\text { possible within most IS - } \\
\text { implementation has not } \\
\text { commenced } \\
\text { The few organizations } \\
\text { who had made big } \\
\text { investments in different } \\
\text { HIS (EPR and pharmacy) } \\
\text { are reporting substantial } \\
\text { returns }\end{array}$ \\
\hline $\begin{array}{l}\text { General Uses } \\
\text { of Web and } \\
\text { Intranet } \\
\text { technology }\end{array}$ & $\begin{array}{l}\text { Limited use as shown by } \\
\text { the following } \\
15 \% \text { - to share clinical } \\
\text { guidelines } 13 \% \text { - to access } \\
\text { multiple databases } \\
\text { simultaneously } \\
33 \% \text { - as a bridge to other } \\
\text { information systems } \\
40 \% \text { - for network wide } \\
\text { communication of any kind }\end{array}$ & $\begin{array}{l}\text { Some early success from } \\
\text { linking "billing and } \\
\text { insurance-query } \\
\text { operations to payers via } \\
\text { the Web" } \\
\text { "Significant interest } \\
\text {...in using the Web to } \\
\text { improve data exchange } \\
\text { with physicians and their } \\
\text { office staff" } \\
\text { About } 50 \% \text { indicated } \\
\text { that they had no plans to } \\
\text { try anything Web-related } \\
\text { in the care-management } \\
\text { area }\end{array}$ & $\begin{array}{l}33 \% \text { - Using existing } \\
\text { clinical and financial } \\
\text { information sources to } \\
\text { construct data repositories } \\
\text { so as to that help spot } \\
\text { trends and improve } \\
\text { decision-making } \\
\text { Further } 22 \% \text { are working } \\
\text { to implement such } \\
\text { practices whilst about } 13 \% \\
\text { plan to start } \\
\text { implementation of similar } \\
\text { activities within a year }\end{array}$ \\
\hline
\end{tabular}


Simultaneously there has been a change in technology (from simple copper wires to optical fibres) via which information is transmitted. This in turn has exponentially increased the bandwidth (Wallace, 1997). The phenomenon of Multimedia has made possible the exchange of information - that can be combined from different formats (sound, video, animation, text and graphics) and presented in an interactive manner. This is fast making multimedia technology the "technology of choice" for the delivery of information (Wallace, 1997).

\section{M-HEALTH : THE TECHNOLOGY FOR HEALTHCARE DELIVERY IN THE FUTURE}

In the healthcare sector, different information technology applications such as clinical information systems, electronic patient records and telemedicine have been used successfully thereby demonstrating their potential to greatly improve the standard of medical care and healthcare administration (Rao, 2001).

Advances in information technology applications have resulted in an "accelerated pace of innovation" (Johns, 1997). Such innovation has resulted in the creation of new opportunities and healthcare concepts such as healthcare information - a term indicating the combined synergistic application of "a science of information, technology, and knowledge...to "health care" (Johns, 1997). All this has led industry experts to predict that, in the near future, healthcare technologies (and, in particular, technologies such as m-health) will be computerized to a considerable extent (Crompton, 2001).

\section{TELEMEDICINE AND M-HEALTH : ORIGINS AND SCOPE}

Telemedicine has been derived from two Greek and Latin words. "Tele" in greek translates as distance while "Mederi" in latin means to heal (Rao, 2001). In a modern context, telemedicine can be stated to be a method of healthcare delivery where advanced video communications technologies are used to bridge the geographical gap that exists between the licensed caregivers and/or the care receiver, so as to provide medical diagnosis and treatment (European Health Telematics Observatory, 1999; Charles, 2000; Nairn, 2001; Garets and Hanna, 1998).

Telehealth has a much broader scope in comparison to telemedicine, as telehealth relates to the bigger issues in healthcare administration and regulation, whilst telemedicine is concerned with the clinical aspects of healthcare delivery (European Health Telematics Observatory, 1999 and Johnson, 2000). Some authors (Nairn, 2001; Noring, 2000) have further delineated between the two by positing that, in telemedicine, healthcare providers fall into the category of physicians whilst, in telehealth, the category of healthcare providers can be extended to include non-physicians, as telehealth encompasses health promotion and disease prevention. 
The earliest documented use of telemedicine can be traced to the 1920 s when radio was employed to link up physicians located on land with ships at sea who were facing medical emergencies. The next leap in telemedicine took place in the 1960s when the US-based National Aeronautics and Space Administration (NASA) pioneered the use telemedicine in space (astronauts had their pulse rates and blood pressure monitored remotely). By the 1970 s, telemedicine additionally took advantage of new emerging satellite technologies (Rao, 2001).

UK, Canadian and Malaysian governments have seized the opportunity to make substantial efforts to link electronically different healthcare centres. In the UK, the Government has committed about USD \$1.4 billion to bring about a transformation in all facets of healthcare. A significant component of this funding is being used in developing a nationwide electronic platform (Crompton, 2001). This high volume of investment worldwide in healthcare technologies has brought about the emergence of several mhealth schemes all over the world (Crompton, 2001; Collins, 2001; The Economist, 1997).

\subsection{Objective of M-Health Applications}

The aim behind any m-health application is to transfer the expertise of the caregiver from one location to another (Johns, 1997). One of the most widely used applications of $\mathrm{m}$-health is teleradiology (use of "image acquisition, storage, display, processing and transport") from one geographical location to another location for diagnosis (Johns, 1997). With advances in technologies, such as telecommunications, multimedia and IT healthcare applications, m-health has the potential to transform the delivery of healthcare permanently.

\subsection{Current M-health Technologies}

The cost of setting up an average m-health station works about to $\$ 50,000$ and incorporates "a computer workstation with 21-inch monitor, electronic stethoscope, ear, nose and throat scope, and an exam camera through which the patients and doctors can see each other"(Tieman, 2000).

There are two main models in m-health: (1) interactive video and (2) store-andforward (Kazman and Westerheim, 1999; Nairn, 2001). The main difference between them is that interactive video allows real-time patient care, whilst the store-and-forward technology is asynchronous (there is a gap between transmission of data and patient care diagnosis). Today, store-and-forward technology applications in m-health include telepathology and teleradiology (Nairn, 2001). The use of email to transmit medical prescriptions by physicians to their patients is fast becoming another major application of store-and-forward technology in m-health (Convey, 2000).

Since store-and-forward technology is asynchronous (communication over telephone lines linking two computers or other peripheral devices using start and stop bits), applications based on this type of technology are being more widely used in comparison to interactive video applications, as they can easily be transmitted over a network. Modern store-and-forward technology applications, in conjunction with advances in telecommunications technologies (such as digital imaging, WAP and fibre optics) are resulting in the creation of a much larger m-health market (Johnson, 2000; Beavan and Frederick, 2000). 
Technologies that offer healthcare videoconferencing as a substrate are still evolving. It is possible to send large amounts of clinical multimedia data (compressed audio and video images) on high speed lines such as broadband technologies over the internet (Nairn, 2001). Given the current pace of advances in internet and videoconferencing technologies, interactive m-health applications will feature heavily in futuristic healthcare systems.

The advantages of $\mathrm{m}$-health include enabling direct links between the caregivers and/or care receivers thereby enabling effective medical care especially to rural populations, saving time and money for caregivers and faster diagnosis and treatment for care receivers (Kazman and Westerheim, 1999; Schooley, 1998; Charles, 2000; Huston and Huston, 2000; Fishman, 1997). Whilst it is clear that $m$-health is more viable compared to traditional telephonic consultations (Sandberg, 2001), in a normal patient care scenario it enables patients to have faster access to alternative specialists and, more importantly, to have access to information about their sickness (Blair, 2001).

One of the major bottlenecks affecting the uptake of m-health in the US is the fact that insurance coverage of $m$-health is generally limited to teleradiology and a few cardiac monitoring procedures (Health Care Strategic Management, 2000). Furthermore in the US, legislation affecting $\mathrm{m}$-health is different in each state.

Another major limitation in $\mathrm{m}$-health is that there is no adequate regulatory structure which addresses such issues as licensure, credentialing, intellectual property and MediCare payments (in the US) (Schooley, 1998; Edelstein, 1999). Governments have started to address these issues with the US Congress taking a pioneering stand in this regard. In 1999, it introduced 22 pieces of legislation relating to m-health (Edelstein, 1999).

\section{THE INTERNET AND M-HEALTH}

An American Medical Association study in 1999 (Swartz, 2000) indicated that 37\% of all physicians in the US were using the Web and by 2000 this figure had risen to about $50 \%$. It has been pointed out that more caregivers have adopted modern ICT applications such as wireless phones and PDAs which allow them to be in contact with patients and, in certain circumstances, to save lives. The use of e-mail by physicians as a method of keeping in touch with patients tripled in less than one year $-10 \%$ of all physicians now use e-mail on a daily or weekly basis to be in contact with their patients (Swartz, 2000).

Initial web-based multimedia patient record systems have been developed which give remote access to telecare providers (Nairn, 2001). We believe that, in the future, webbased multimedia patient administration systems will become the norm for m-health. A similar concept has been put forward by the National Health Service (NHS) in the UK, where healthcare institutions are being asked to adopt an Electronic Patient Record (EPR) system at six varying levels of implementation (NHS, 1998).

One of the biggest indicators that portends the rise of m-health has been the ruling by the Federal Communications Commission (FCC) in the US who had recently cast "....an historic vote dedicating a portion of the radio spectrum for wireless medical telemetry devices such as heart, blood pressure and respiratory monitors" (Health Management Technology, 2000, p12 ). 


\subsection{Mobile Computing and Workflow}

In the near future, third generation technologies such as Code Division Multiple Access (CDMA) 2000 are expected to raise the transmission standard to about 2.0 Mbps (Cancela, 2001). When this happens, it is quite likely that the vision of an "integrated voice, data, video technology" (Cancela, 2001) will become a reality which is likely to have a significant impact on the use of $m$-health.

The success of second-generation wireless networks has led to an explosion in the use of wireless applications to transfer voice and data services. This has raised the possibility that future wireless networks might support cost-effective broadband multimedia services (Pinto and Rocha, 1999). Technologies such as Wireless Application Protocol (WAP) have enabled patients and doctors to remain in closer contact. There are successful WAP-based products (such as LifeChart.com) through which doctors can monitor online their patient's condition, and take care of their healthcare needs (Purton, 2000).

Another example of the use of WAP is WirelessMed, through which UK Doctors have wireless access to clinical data on Medline, the largest US government database comprising more than 12 million medical references (which supports download speeds of up to 400-words in a few seconds). Another example of WAP-enabled healthcare products is MedicinePlanet which aims to bring local health information (health news, current health alerts, details on prevailing healthcare systems) to travellers using mobile phones (Purton, 2000). WAP technology is facing strong competition from other medical wireless systems based around PDA (Personal Digital Assistant) platforms which support downloads of a standard patient image in 10-15 seconds (Parkes, 2001).

The main disadvantages of mobile computing (limited battery and processor power) should diminish as new technologies, allowing higher bandwidths, are introduced onto the market (Satyanarayanan, 1996). Workflow-based applications, in conjunction with mobile computing technologies such as WAP and PDA, have the potential to transform the delivery of healthcare information.

Modern day IT applications in healthcare use protocols centered on Mobile Computing and the Internet. Some of them such as Wireless Local Area Network (WLAN) have already demonstrated their potential and financial viability. WLAN-based mobile computing allow healthcare workers to interact in real-time with the hospital's host computer system to enter, update and access patient data and associated treatment from all clinical departments. All this is possible not only from the patients' bedside, but also from a number of geographical locations within the hospital where the WLAN is installed.

The fact that a WLAN takes about one hour to be made operational has been trumpeted as one of the biggest advantages in comparison to a more traditional network, installation of which would take significantly longer (McCormick, 1999). It has been found that the average pay-back period for the initial costs of WLAN installations is 8.9 months. In a survey of WLAN healthcare installations, $97 \%$ of customers indicated that "WLANs met or exceeded their expectation to provide their company a competitive advantage" and that "if the productivity benefits are measured as a percentage return on the total investment ... the return works out to be 48\%" (McCormick, 1999, p13). 
The use of Personal Digital Assistant (PDA) by physicians has witnessed rapid acceptance in recent times. About $40 \%$ of all physicians currently use PDAs (Serb, 2002). However, the majority of physicians are using PDAs to perform static functions. Most of them use PDAs to collect reference material with ePocrates - a drug reference application that can assist physicians in looking up drugs by name or diagnoses, crossreference analogous medications or generic alternatives and obtain alerts on interactions. This application has been categorised by the US based Journal of the American Medical Association to be indispensable (Serb, 2002).

A few pioneering physicians have started to use PDAs in an interactive way i.e. to write prescriptions, to keep a record of all daily clinical patient interactions and for bedside charting. The financial viability of PDAs have been demonstrated by a pilot study which has shown that for every US \$1 that was invested in PDAs, the return in the form of lower administrative costs was over $\$ 4$ (Serb, 2002).

\section{FINANCIAL ESTIMATES FOR M-HEALTH}

Estimates for worldwide m-health services suggests that the market is valued at $\$ 2.5$ billion a year (Surry, 2001). Other estimates for 2002 project the US market alone to be around \$3 billion - a big leap from \$65 million in 1997 (Industrial Robot, 1998).

A study by Waterford Telemedicine Partners Inc in Feburary 2000 has indicated that $\mathrm{m}$-health is projected to have an annual growth rate around $40 \%$ in the first decade of the 21 st century. The study predicts that, by the year 2010 , m-health will account for at least $15 \%$ of healthcare expenditure (Health Care Strategic Management, 2000).

M-health technologies, in conjunction with state-of-the-art Electronic Health Record (EHR) systems, are changing the face of healthcare. M-health technologies have the potential to replace $5 \%$ of hospital stays, $5 \%$ of nursing home care, and $20 \%$ of home health visits (Fishman, 1997), resulting in savings of time and money for both patients and doctors. Additionally, caregivers have more time to devote to clinical activities such as medical diagnosis and treatment. A study on the medical reimbursement of m-health applications indicates that "telemedicine for radiology, prisoner health care, psychiatry, and home health care are the most cost effective applications ...that are paid for by insurers" (Charles, 2001, p66).

\section{WORLDWIDE APPLICATIONS OF M-HEALTH}

One of the most widely used applications of m-health is teleradiology (Rao, 2001). The use of m-health applications to monitor patients has been recommended in asthma, congestive heart failure and for diabetes (Friedewald Jr, 2001).

A study carried out by the Agency for Healthcare Research and Quality in the US identified 455 telemedicine programs worldwide out of which 362 are being used in the US (Trembly, 2001). The study has indicates that $m$-health is most commonly used for: 1] Consultations or second opinions (performed in 290 programs), 2] Diagnostic test interpretation (169), 3] Chronic disease management (130), 4] Post-hospitalization or post-operative follow-up (102), 5] Emergency room triage (95), 6] Visits by a specialist (78) and that about 50 programs provide services in patients' homes. 
Countries such as Malaysia have already integrated m-health with the electronic health record concept and there is a national telemedicine strategy in place. Teleconsultations are being carried out on a regular basis in Malaysia. In Sweden, mhealth is being used to reduce the stay of children in hospital. This is being achieved by using local telecommunications to connect to the health monitoring equipment (for heart rate, rhythm and blood pressure) and is installed at the residence of the patient (Collins, 2001). In the UK, at the West Surrey Health Authority, patients who are regarded as potential heart failures are monitored electronically for 24 hours a day at their residences (Crompton, 2001).

A report by the US based Association of Telehealth Service Providers has indicated that in 1996 there were about 22,000 telemedicine consultations, which rose to 42,000 in 1997 and by 1998 to about 58,000 (Tieman, 2000).

\subsection{True Life Examples that Validate M-Health Applications}

In the UK, the North Manchester primary care group has used telemedicine applications to reduce the "average waiting time for a dermatology appointment from 18 months to 17 days" (Cross, 2000). In the US, m-health is said to have had tremendous benefits in reducing hospital intensive care costs (ICU). Each day in ICU costs on average $\$ 2,500$ to the insurance company, which can be reduced to $\$ 35$ by a routine teleconsultation (Cross, 2000).

The use of m-health can significantly aid patients in the battle to combat diabetes whilst reducing the associated expenditure. It has been estimated that the use of $m$-health can save the US government, about " $\$ 247$ million per year through early intervention and nearly double that if telemedicine can extend the reach of treatment" (Blair, 2001, p4).

In Cornwall (UK) a pilot telemedicine project for teledermatology was undertaken. General Practitioners (GPs) from three surgeries (medical centers) used to send pictures of skin conditions from Cornwall to the county's two consultant dermatologists. They would then make an assessment as to whether the patient was required to visit the dermatologists for treatment or whether the GP could treat them in Cornwall itself. This m-health application found that "one in four patients did not need to see a specialist and could be treated by their family doctor" and reduced the workload of the two overworked dermatologists (The Guardian, 2001).

In 2001, a telemedicine application was used for the first time to carry out telesurgery. Doctors via computer from New York operated on a 68-year-old woman in Strasbourg, France to remove her gall bladder (Alpert, 2001; Johnson, 2002). The patient was released from the hospital 48 hours after surgery and recommenced regular activities the following week (Johnson, 2002). 
Telemedicine has been used very successfully in the US state of Arizona. Due to the geographical nature of Arizona, it is not possible for its residents which number in thousands to have immediate access to leading edge healthcare, as closet centres are exist 150 miles away or more (Health Management Technology, 2001). The Arizona Telemedicine Program today has more then 96 telephysicians representing 60 medical sub-specialities such as teleradiology, teledermatology, telepsychiatry, telecardiology, teleorthopedics, teleneurology and telerheumatology. These telephysicians have seen more than 11,000 patient cases and have provided primary diagnoses, expert consultation and provision of second opinions. Another pioneering advantage of telemedicine in Arizona has been to decrease risky travel, especially for patients with unstable medical conditions (Health Management Technology, 2001).

The Arizona Telemedicine Program has resulted in significant cost saving for healthcare delivery as the average cost "for a rural patient's visit to an urban health center has dropped from $\$ 520$ to $\$ 105$ " and there exists significant potential in lowering "the average cost for routine home health visits ... from \$140 to as low as \$55 per visit". One of the more significant achievements of the Arizona Telemedicine Program was to reduce healthcare expenditures in prisons, with the average cost for a prison inmate's healthcare visit falling from \$415 to \$140 (Health Management Technology, 2001, p47).

One of the most important technological enablers that is likely to affect transmission of healthcare information and healthcare delivery is the connectivity via satellite technology. It was estimated that there were about 150 satellites in orbit in 2001 (Rao, 2001). More significant is the fact that about " 1,700 commercial satellites are scheduled for launch in the next decade worldwide" (Rao, 2001, p227). This could be the technological catalyst that brings about a transformation in the manner how healthcare delivery takes place, particularly in those areas where there is a geographical gap between the caregivers and carereceivers or where there is a substantial time constraints on specialized caregivers. Tele-consultations between caregivers and carereceivers and between themselves could see a likely increase (Rao, 2001).

\section{IMPORTANT INNOVATIONS THAT HOLD GREAT POTENTIAL TO BE REVOLUTIONARY CHANGE CATALYSTS FOR THE HEALTHCARE SECTOR}

\subsection{Biomedical Knowledge: The Untapped Potential in Healthcare}

Advances in technology have been the hallmark of the healthcare sector, particularly with regard to advances in biomedical sciences. Today there are " 10,000 known diseases, 3,000 drugs, 1,100 lab tests, 300 radiology procedures, 1,000 new drugs and biotechnology medicines in development and 2,000 individual risk factors" (Pavia, 2001, p12). This has had an enormous impact on healthcare and, in particular, has rendered the concept of an expert in a particular domain in healthcare irrelevant as shown above it is not possible for one human being to have all the relevant knowledge in their domain of speciality (Pavia, 2001; Rockefeller, 1999). 
For example today, "Organ and tissue scanning speed is doubling every 26 months, making tests both faster and cheaper...Image resolution is doubling every 12 months...the increase in computer power (four-fold over the next 20 years) and the availability of inexpensive bandwidth" (Pavia, 2001, p12). All of the above is likely to change our own perception of what information is available and even possible for one human being to acquire.

\subsection{The Genetic Engineering (un)revolution}

Advances in modern day genetic sciences have increased the number of potential drug compositions from a mere 400 to over 4,000 (Pavia, 2001). This has occurred despite the fact that the rate of adoption of computer applications in healthcare is slower in comparison to other industries (Johns, 1997). The pace of discovery of new drugs may well undergo an exponential leap when the above observation is seen in context of the forecasted increase in computing power as discussed previously in this chapter.

Perhaps the biggest tragedy in the history of modern science was the fact that the announcement regarding the completion of the Human Genome project did not create any ripples in the mindset of healthcare decision-makers and academics or propel a new wave of healthcare discoveries (Jones, 2001). We hypothesize that this situation is not likely to prevail for much longer. The impact of the completion of the project will profoundly change the concept of healthcare itself within the next 25 years (Jones, 2001).

Unfortunately, the contemporary focus in m-health is only on how best to disseminate the information, which could be fatal for the future of telehealth and $\mathrm{m}$ health. Rather than creating or disseminating contextual knowledge, m-health applications are being used to disseminate data and/or information. Futuristic m-health schemes would need to support the transfer of information with context (i.e., such schemes would have to become dynamic in nature). One of the big drawbacks of $\mathrm{m}$ health is that most systems force the caregiver specialist to look at medical issues in isolation, whereas more detailed information (such as the patient's medical history) might help in arriving at a better informed medical diagnosis (Nairn, 2001).

\section{DISCUSSION}

This section summarises how m-health will affect healthcare delivery. It is argued that $\mathrm{m}$-health will alter healthcare delivery in the following ways:

(a) It would be important to consider having a definition of what is meant by the term "healthcare". This is by no means an easy task. We believe that m-health will reduce both critical and non-critical healthcare treatments. However, in the immediate future mhealth is likely to reduce the cost of routine consultations. Advances in technology will help patients carry out routine medical tests, reducing the number of visits to the physician, thereby reducing costs for routine consultations. However there could be an increase for non-routine expenditure for complex treatments.

(b) m-health applications such as teleradiology will increase. In addition, m-health will be used increasingly for the following purposes: consultations or second opinions, diagnostic test interpretation, post-hospitalization or postoperative follow-up, emergency room triage and televisits. The use of m-health applications to monitor patients will see an increased use (eg. for asthma, congestive heart failure, diabetes). 


\subsection{Need for Incorporating Organisational Perspectives in M-Health Processes}

The last two decades of the twentieth century has witnessed a shift in the concept of healthcare information. This shift has been marked by the coming of age of paradigms such as $\mathrm{m}$-health, bioinformatics, biomedical and genetic engineering resulting in exponential advances in healthcare knowledge (Dwivedi et al, 2002). One of the most significant implications of this shift has been the realization that the applications of IT advances in the healthcare sector have caused an information explosion. Individual healthcare stakeholders are not going to be in position to adapt to the above with ease. Any solution would call for significant integrated technological support in the human healthcare decision-making process (Dwivedi et al, 2001a; 2001b; 2002).

It has been proposed that holistic health will emerge as an alternative to complement traditional medicine (Church et. al, 1996; Dervitsiotis, 1998). As patients' homes become the homebase for delivering more and better types of care, people will expect "King Quality, Queen Value" (Nelson, Batalden and Mohr, 1998, p3). Healthcare organisations need to be fully aware of the organisational implications of telehealth initiatives.

The technology associated with $\mathrm{m}$-health schemes transcends geographical, institutional and disciplinary boundaries. M-health redefines organisational roles and responsibilities and by disseminating knowledge and information, it allows healthcare professionals and patients to relate to each other. The astonishing rate of change makes strategic planning extremely difficult.

Appreciating the role of management and how it controls and monitors resource requirements needs is crucial. Having identified suitable individuals and jobs, it must be emphasized that $\mathrm{m}$-health is an additional health-delivery avenue and no healthcare provider should be forced to use the new technology. M-health delivery may be better suited to people who tend to exhibit such traits as a greater ability to structure their workday, more efficiently separate work and family life, or those whose jobs are more independent and proactive.

\subsection{Knowledge Management}

Knowledge management $(\mathrm{KM})$ is considered as a source of great competitive advantage (Nonaka, 1991; Wiig, 1994). Knowledge can either be tacit or explicit (Beijerse, 1999; Gupta, Iyer and Aronson, 2000; Hansen, Nohria, and Tierney, 1999). Explicit knowledge typically takes the form of company documents and is easily available, whilst tacit knowledge is subjective and cognitive. The ultimate objective of $\mathrm{KM}$ is to transform tacit knowledge into explicit knowledge to allow effective dissemination (Gupta, Iyer and Aronson, 2000).

Knowledge Management and Information and Communication Technologies (ICT) as disciplines do not have commonly accepted or de-facto definitions. However some common ground has been established which covers the following points.

KM is a multi-disciplinary paradigm (see Figure 1) which uses technology to support the acquisition, generation, codification and transfer of knowledge in the context of specific organisational processes. ICT refers to the recent advances in applications of communication technologies that have enabled access to large amounts of data and information when seeking to identify problems or solutions to specific issues (Dwivedi et al, 2001a). 


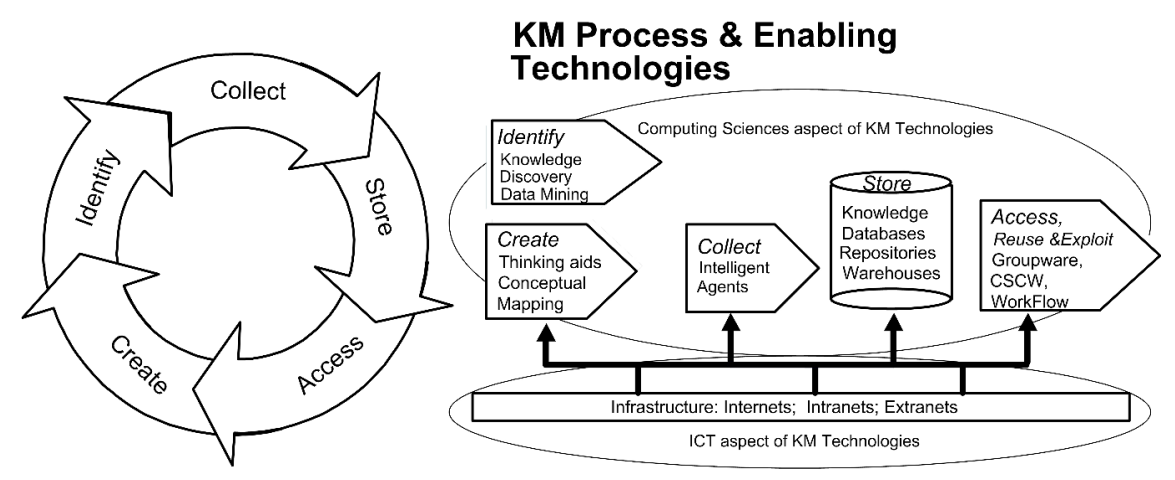

Figure 1. KM Process and Enabling Technologies. Adapted from (Skyrme, 1999)

\subsection{Impact of KM on M-health}

There are many factors which may influence m-health which can be reduced to three fundamental areas: technological, business and social (Bali, 2000b). These areas all consider the current pace of technological change. The commercial environment is undergoing a period of accelerated information technology change, which some would argue is a revolution. Developments in technology, social considerations, government fiscal policy and business aims and objectives need to be fully understood in order to fully exploit the social and economic benefits that are emerging as a result of m-health (Bali and Naguib, 2001). Healthcare organisations are in a constant state of change and $\mathrm{m}$-health is both a key manifestation and enabler of this change. However, researchers and practitioners need to appreciate the implications and ramifications of such a change.

The multi-disciplinary aspect of KM research has resulted in a multitude of models and approaches, all of which look at KM from perspectives similar to m-health. KM is viewed as a methodology involving the interaction between people, culture, information technology, and organisations. A different perspective discusses KM's relationships between culture, content, process, and infrastructure. Another approach reflects that a successful KM programme must bring together political, organisational, technical and cultural organisational aspects (Bali, 2000a; Puccinelli, 1998; Chait, 1999; Havens and Knapp, 1999)

We would like to establish the interrelationship between KM and m-health by stating that both have been brought about by the ICT revolution, and that both are bringing about fundamental changes which are redefining the work place of contemporary healthcare organisations. Another common point is that both $\mathrm{KM}$ and $\mathrm{m}$-health are concerned with dissemination of information in a manner which ensures that information is available when required. 
We believe that the difference between KM applications and m-health applications lies in the application of ICT. As compared to KM, ICT in telehealth and telemedicine is in its relative infancy. Unfortunately, the contemporary focus is only on how best to disseminate the information - which could be fatal for the future of m-health (i.e., current use is static). Rather than creating or disseminating contextual knowledge, m-health applications are being used to disseminate data and or information. Futuristic m-health schemes would have to support the transfer of information with context (i.e., such schemes would have to become dynamic in nature).

One of the big drawbacks of m-health is that most systems force the caregiver specialist to look at medical issues in isolation, whereas more detailed information (such as the patient's medical history) might help in arriving at a better informed medical diagnosis (Nairn, 2001). Initial web-based multimedia patient records systems have been developed, which give remote access to the telecare providers (Nairn, 2001). We believe that, in the future, web-based multimedia patient administration systems will become the norm for m-health. A similar concept has been put forward by the NHS (National Health Service) in the UK, where healthcare institutions are being asked to adopt an Electronic Patient Record (EPR) system at six varying levels of implementation (NHS, 1998).

Healthcare institutions require a framework which would help assess how best to identify and create knowledge from internal and external organisational experiences and how best to disseminate these on an organisation-wide basis. This would call for the contextual recycling of knowledge which has been acquired from the adoption of $\mathrm{m}$ health trials. KM can assist $\mathrm{m}$-health to become viable by giving healthcare information context, so that other healthcare providers can use m-health to extract knowledge and not information.

For this to happen, futuristic m-health systems would have to shift their emphasis to deal with the intangibles of knowledge, institutions and culture and that the KM paradigm is aptly suited for this role. This is due to the fact that one of the important reasons behind the emergence of the KM concept is that, even though our access to data and information has increased exponentially, our capability to acquire knowledge (by giving the information context) has not become an industry-wide reality. This also holds true for the healthcare industry.

\section{THE NEED FOR A KM FRAMEWORK FOR M-HEALTH}

Healthcare managers are being forced to examine the costs associated with healthcare and are under increasing pressure to discover approaches that would help to carry out activities better, faster and cheaper (Dwivedi et al, 2001b; Latamore, 1999). For this to happen, the m-health sector needs to shift it's emphasis to deal with the intangibles of knowledge and culture (Dwivedi et al, 2001a). Healthcare institutions (HIs) adopting mhealth applications would require a KM framework which, in light of their ICT implementation level, would assist in the discovery and creation of new knowledge (see Figure 2). 


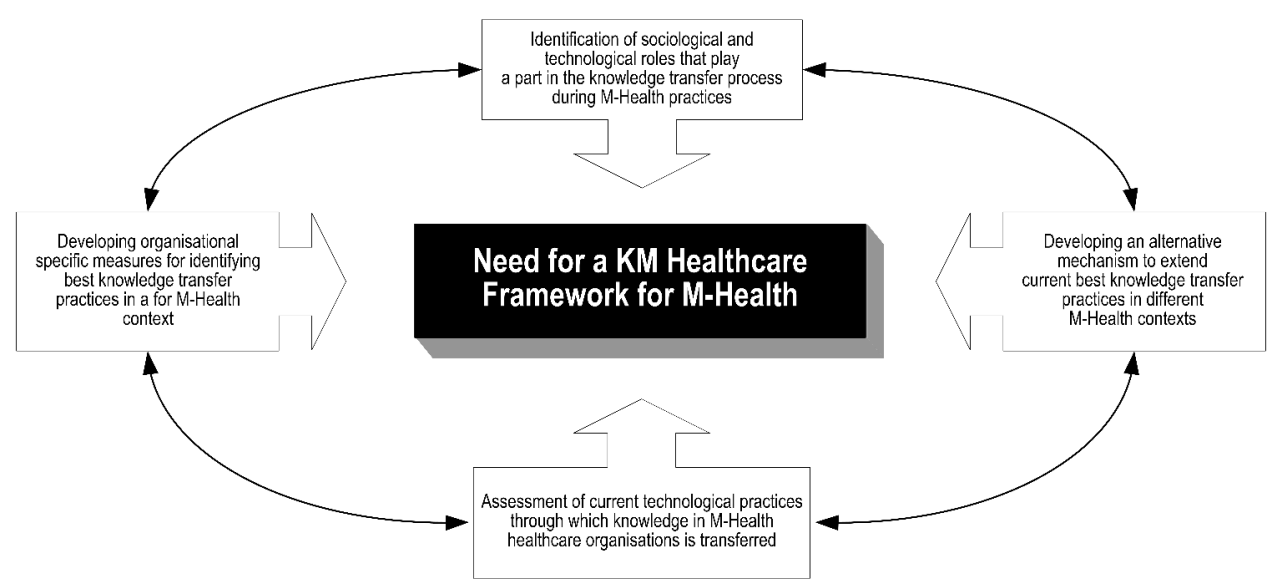

Figure 2. Need for a KM Framework for the m-health sector

HIs need to identify key sociological and technological roles affecting the knowledge-transfer process and to develop organizational-specific measures for identifying best knowledge transfer practices (see Figure 2).

Any KM strategy that is being proposed should extend best knowledge transfer practices on an organization-wide basis. Our contention above has been confirmed by the Canadian Department of Health (Health Canada, 2001). KM can assist healthcare institutions to become viable by giving healthcare information context, so that other healthcare providers can extract knowledge and not information (Dwivedi et al, 2001a). The cornerstone of this chapter is that the KM paradigm can enable the healthcare sector to successfully overcome the information and knowledge overload in healthcare (Dwivedi et al, 2002).

\section{CONCLUSIONS}

We have discussed important technologies that are driving forces in healthcare and have considered the implications of their advances on healthcare in general. We contend that if the impact of these healthcare technologies are seen together, then the conclusion from a healthcare informatics perspective is clear. In the future, m-health systems would have an increased interest in knowledge recycling of the collaborative learning process acquired from previous $\mathrm{m}$-health practices. 
Twenty first century clinical practitioners need to acquire proficiency in understanding and interpreting clinical information so as to attain knowledge and wisdom whilst dealing with large amounts of clinical data. This would be dynamic in nature and would call for the ability to interpret context-based healthcare information. This challenge cannot be met by an IT led solution. The solution needs to come from a domain that supports all the three integral healthcare system components (i.e. people, processes and technology) of the future. We believe that the KM paradigm can offer solutions to healthcare institutions, allowing them to face the challenge of transforming large amounts of medical data into relevant clinical information by integrating information using workflow, context management and collaboration tools, and give healthcare a mechanism for effectively transferring the acquired knowledge, as and when required.

\section{REFERENCES}

Alpert, M. (2001), “Surgeons without borders," Scientific American, volume.285, issue.5, pp.28.

Applebaum S.H. and Wohl, L. (2000), "Transformation or change: some prescriptions for health care organizations," Managing Service Quality, volume.10, no.5, pp. 279-298.

Bali, R.K. (2000a), "Towards a qualitative informed model for EPR implementation: considering organizational culture," Proc of the IEEE Int Conf on Information Technology Applications in Biomedicine (ITAB-ITIS) Arlington, USA, pp.353-358.

Bali, R.K. (2000b), "Organizational and social impacts of telehealth: a cause for concern," Proc of the IEEE Int Conf on Information Technology Applications in Biomedicine (ITAB-ITIS), Arlington, USA, pp.54-59.

Bali, R.K. and Naguib, R.N.G. (2001), "Towards gestalt telehealth: considering social, ethical and cultural issues," [CD-ROM] IEEE Canadian Conference on Electrical and Computer Engineering, Toronto, Canada.

Beavan, B. and Frederick, J. (2000), “Telemedicine raises some points to ponder," Defense Counsel Journal, volume.67, issue.3, pp. 400-403.

Beijerse, P.uit.R. (1999), "Questions in knowledge management: defining and conceptualising a phenomenon", Journal of Knowledge Management, volume. 3, issue. 2, pp. 94-109.

Blair, R. (2001), "Slice of life," Health Management Technology, volume.22, issue. 2, pp. 4.

Cancela, T.A. (2001), "Promises to keep," "Health Management Technology, ”volume.22, issue.4, pp. 38-40.

Chait, L.P. (1999), "Creating A Successful Knowledge Management System." The Journal Of Business Strategy, volume. 20, issue. 2, pp. 23-26.

Charles, B.L. (2000), "Telemedicine can lower costs and improve access," Journal of the Healthcare Financial Management Association, volume.54, issue. 4, pp. 66-69.

Church, A.H., Siegal, W., Javitch, M., Waclawski, J., and Burke, W.W. (1996), "Managing organizational change: what you don't know might hurt you," Career Development International, volume.1, no.2, pp. 2530 .

Collins, J. (2001), "So far and yet so near with telemedicine," [1DD Edition], The Times, London (UK), Jan 9, pp. 2.

Convey, M.C. (2000), “Telemedicine concerns healthcare risk mgrs” National Underwriter, volume.104, issue. 46, pp.8.

Crompton, S. (2001), "Virtual hospital speeds recovery," [Final 1 Edition]. The Times, London (UK), Jun 5., pp. 2 .

Cross, M. (2000), "Society: "Lives online: Tony Blair has committed the government to an electronic revolution of its care programme by 2005 . Is this realistic? Can IT really change the way we deliver public services?," The Guardian, Manchester (UK), Nov 15, pp. 2.

Dervitsiotis, N.K. (1998), "The challenge of managing organizational change: exploring the relationship of reengineering, developing learning organizations and total quality management," Total Quality Management, volume.9, no.1, pp.109-22.

Dwivedi, A., Bali, R.K., James, A.E. and Naguib, R.N.G. (2001a), "Telehealth Systems: Considering Knowledge Management and ICT Issues", Proc. of the IEEE-EMBC 23rd Annual International Conference of the IEEE Engineering in Medicine and Biology Society (EMBS), Istanbul, Turkey, pp. 3614-3617. 
Dwivedi, A., Bali, R.K., James, A.E. and Naguib, R.N.G. (2001b), "Workflow Management Systems: the Healthcare Technology of the Future?", Proc. of the IEEE EMBC-2001 23rd Annual International Conference of the IEEE Engineering in Medicine and Biology Society (EMBS), Istanbul, Turkey, pp. 3887-3890.

Dwivedi, A., Bali, R.K., James, A.E., Naguib, R.N.G. and Johnston, D. (2002), "Merger of Knowledge Management and Information Technology in Healthcare: Opportunities and Challenges", Proc. of the IEEE Canadian Conference on Electrical and Computer Engineering (CCECE) 2002. Winnipeg, Canada, volume.2, pp.1194-1199.

Edelstein, S.A. (1999), "Careful telemedicine planning limits costly liability exposure," Journal of the Healthcare Financial Management Association, volume.53, issue. 12, pp. 63-69.

European Health Telematics Observatory, (1999), "Draft International Convention on Telemedicine and Telehealth," Medicine and Law committee of the International Bar Association, http //www.ehto.org/ legal /draftconvention.doc.

European Telework Organization, (1999), "Status report on European telework," New Methods of Work.

Feldman, D. and Gainey. T. (1997), "Patterns of telecommuting and their consequences: framing the research agenda", Human Resource Management Review, volume.7, No.4, pp.369-388.

Fishman, D.J. (1997), "Telemedicine: bringing the specialist to the patient," Nursing Management, volume.28, issue. 7, pp.30-32.

Friedewald Jr, V.E. (2001), "Returning home,” Health Management Technology, volume.22, issue.9, pp. 22-27.

Garets, D. and Hanna, D. (1998), "Emerging managed care technologies," Health Management Technology, volume.19, issue. 11 , pp. 28-32,

Gupta, B., Iyer, L.S. and Aronson, J.E. (2000), "Knowledge management: practices and challenges", Industrial Management \& Data Systems, volume. 100, issue. 1, pp. 17-21.

Hansen, M., Nohria, N. and Tierney, T. (1999), "What's your strategy for managing knowledge?," Harvard Business Review, volume.77, issue. 2, pp. 106-116.

Havens, C. and Knapp, E. (1999), "Easing Into Knowledge Management”, Strategy \& Leadership, volume. 27, issue. 2, pp. 4-9.

Health Canada, (1998), "Vision and Strategy for Knowledge Management and IM/IT for Health Canada" (WWW document), Available from: http://www.hc-sc.gc.ca/iacb-dgiac/km-gs/english/vsmenu_e.htm. [Accessed March 20, 2001].

Health Care Strategic Management. (2000), "Telemedicine will grow 40\% annually, help health systems create 'centers of excellence'," Health Care Strategic Management, volume. 18, issue.2, pp. 5.

Health Management Technology. (2000), "Advice from the American Hospital Association," "Health Management Technology, " volume.21, issue.10, pp. 12.

Health Management Technology. (2001), "Powering the Arizona telemedicine program," volume.22, issue.6, pp. 46-47.

Huston, T.L. and Huston, J.L. (2000), "Is telemedicine a practical reality?. Association for Computing Machinery", Communications of the ACM, volume. 43, No. 6, pp.91-95.

Industrial Robot. (1998), “Clinical telemedicine to expand by 2002,” Industrial Robot, volume. 25 no. 3, pp. 15.

Johns, P.M. (1997), "Integrating information systems and health care", Logistics Information Management, volume.10, No.4, pp.140-145, viewed 17 October 2002, ProQuest database ABI/INFORM, 86066571.

Johnson, D. (2002), “The telesurgery revolution," The Futurist, volume.36, issue.1, pp. 6-7.

Johnson, D.E.L. (2000), "Telehealth expands unrealized dream," Health Care Strategic Management, volume.18, issue.6, pp. 2-3.

Jones, W.J. (2001), "Genetics: Year zero", Health Forum Journal, volume. 44, issue. 6, pp. 14-18.

Kazman, W. and Westerheim, A.A. (1999), "Telemedicine leverages power of clinical information", Health Management Technology, volume. 20, No. 9, pp. 8-10.

Latamore, G.B. (1999), "Workflow tools cut costs for high quality care", Health Management Technology, volume. 20 , issue. 4 , pp. 32-33.

Marien, M. (1989), "IT: you ain't seen nothin yet" in T Forester ed. Computers in the Human Context, Basil Blackwell, Oxford, pp. 7-41.

McCormick, J. (1999), "Wireless hospitals: New wave in healthcare technology," Health Management Technology. volume.20, issue.6, pp. 12-13.

Moran, D.W. (1998), "Health information policy: on preparing for the next war," Health Affair, volume.17, issue. 6 , pp. 9-22.

Morrissey, J. (2000), "Internet dominates providers' line of sight," Modern Healthcare, volume.30, issue. 15, pp. 72-92.

Morrissey, J. (2001), "Wanting more from information technology," Modern Healthcare, volume.31, issue.6, pp. $66-84$.

Morrissey, J. (2002), "High on tech, low on budget," Modern Healthcare, volume.32, issue.4, pp. 57-72. 
Nairn, G. (2001), “Technology pulls together medicine's diagnostic tools: telemedicine," Financial Times, London, Feb 21, pp. 9.

Nelson, C.E., Batalden, P.B. and Mohr, J.J., (1998), "Building a quality future," Frontiers of Health Services Management, volume.15, no.1, pp 3.

NHS. (1998), "Overview of benefits from electronic patient records," http // www.nhsia.nhs.uk/ strategy/ full/2.htm.

Nonaka, I. (1991), “The Knowledge-Creating Company”, Harvard Business Review, volume. 69, issue. 6, pp. 96-104.

Noring, S. (2000), "Telemedicine and telehealth: principles, policies, performance, and pitfalls," American Journal of Public Health, volume. 90, issue. 8, pp. 1322.

Parkes, S. (2001), "Medicine may be one of Wap technology's 'killer' applications: mobile services for doctors," Financial Times, London, Feb 21, pp. 04.

Pavia, L. (2001), "The era of knowledge in health care", Health Care Strategic Management, volume. 19, issue. 2 , pp. $12-13$.

Pinto, P. and Rocha, R. (1999), "Trends on wireless systems radio and satellite," European Health Telematics Observatory, http // www. ehto.org / projects/ trends.html.

Puccinelli, B. (1998), "Strategies for sharing knowledge", Inform, volume. 12, issue. 9, pp. 40-41.

Purton, P. (2000), "Good prognosis for mobile health services: medical applications," Financial Times, London, Sep 20, pp. 24.

Rao, S.S. (2001), "Integrated health care and telemedicine," Work Study, volume.50, no.6 pp. $222-228$.

Rockefeller, R. (1999), "Informed shared decision making: Is this the future of health care?" Health Forum Journal, volume. 42 , issue. 3 , pp. 54-56.

Sandberg, L.A. (2001), "The pediatric promise," Health Management Technology, volume.22, issue.2, pp. 4647.

Satyanarayanan, M. (1996), "Mobile information access," IEEE Personal Communications, volume.3, no.1, pp 26-33.

Schooley, A.K, (1998), "Allowing FDA regulation of communications software used in telemedicine: a potentially fatal misdiagnosis", Federal Communications Law Journal, volume. 50, issue. 3, pp. 731-751.

Serb, C. (2002), "Health care at your fingertips?," Hospitals \& Health Networks, volume.76, issue.1, pp. 44-46.

Sherer, J. (1995), "The human side of change," Healthcare Executive, volume.12, no.4, pp.8-14.

Skyrme, D.J. (1999), Knowledge Networking: creating the collaborative enterprise, Butterworth-Heinemann, Oxford.

Stanworth, C. (1998), "Telework and the information age", New Technology, Work and Employment, volume. 13, No.1, Mar 1998, pp.51-62.

Surry, M. (2001), "The cyber medics," Asian Business, volume. 37, issue.10, pp. 46-47.

Swartz, N. (2000), "Wireless house calls," Wireless Review, volume.17, issue.24, pp. 48-53.

The Economist. (1997), "Big sister is watching you: telemedicine,", volume.342, issue.7999, pp. 27 , Jan 11.

The Guardian. (2001), Society: frontline: "How skin specialists became west country pioneers," The Guardian, Manchester (UK), Aug 1, pp. 10.

Tieman, J. (2000), "Monitoring a good opportunity," Modern Healthcare, volume.30, issue.43, pp. 75-80, viewed 26 September 2002, ProQuest database ABI/INFORM, 62701984.

Trembly, A.C. (2001), "Federal study backs use of telemedicine," National Underwriter, volume.105, issue.19, pp. 13.

Wallace, S. (1997), "Health information in the new millennium and beyond: the role of computers and the Internet," Health Education, no. 3, pp. 88-95.

Wiig, K.M. (1994), "Knowledge management: the central focus for intelligent-acting organizations," Schema Press. 